\title{
NSF programs in the behavioral and neural sciences
}

\author{
FRED STOLLNITZ \\ National Science Foundation, Washington, D. C. 20550
}

\begin{abstract}
The National Science Foundation's new Division of Behavioral and Neural Sciences supports basic research and related activities in psychology, ethology, anthropology, linguistics, and the neurosciences. Research equipment and facilities can be provided either through a grant supporting a research project or through a separate grant. The scientific importance and soundness of the research to be conducted, as evaluated by reviewers from the research community, are the main criteria for support. Preparation of proposals is briefly discussed, and sources of further information are noted.
\end{abstract}

The National Science Foundation's new Division of Behavioral and Neural Sciences includes six programs: Neurobiology, Sensory Physiology and Perception, Psychobiology, Social Psychology, Anthropology, and Linguistics. (Early in 1976, plans were made to add a Memory and Cognitive Processes Program and to broaden the Social Psychology Program to Social and Developmental Psychology.) Among them. these programs offer support for basic research in all areas of psychology and in other behavioral sciences. Except for clinical studies, proposals for applied research can also be considered, but the chief criterion for evaluation is always the expected contribution of the research to fundamental scientific knowledge.

Each proposal is sent to several reviewers selected for their expert knowledge of the research topic of the proposal. In addition, most proposals are reviewed by one of our advisory panels, whose members represent a broad range of research areas. You can learn the current membership of each panel from the corresponding program, but the names of reviewers of a particular proposal are not disclosed. However, verbatim copies of reviews requested by NSF after January 1, 1976, will be available to the principal investigator - a procedure which I hope will enable us to give applicants more helpful comments more promptly than we have sometimes been able to in the past.

Available funds are severely limited, so only the most meritorious new and continuing projects can be supported. The proposal should be written in a way that makes the importance of its scientific contribution clear to all reviewers, including those whose own research may be in different areas. The proposal should present your research plan in enough detail to permit evaluation of its merit, your competence, and the time and budget required for the project. However, reviewers appreciate concise proposals. Reprints or preprints may help to present needed details; cite them clearly in the text of the proposal and send 20 copies (attached to the proposal copies) if you wish all reviewers to see them.
Just as we rely very heavily on reviewers' judgments of scientific merit, we also ask for comments on the proposed budget. For example, reviewers who use computers in their own research can give us helpful guidance on the need for computer time or equipment for a proposed project. If the equipment is needed for a highly meritorious project, we will provide it assuming that we have sufficient funds to support the project. If the institution can share the cost of the equipment, it helps in several ways: Our limited funds can support more projects; it helps to demonstrate a real need for the equipment; it reflects a recognition that the institution will continue to own the equipment even after the project ends; and it may indicate a willingness to make the equipment available to other researchers besides the applicant and his collaborators.

Besides grants for research projects and for related activities, we can make grants for research equipment and faclities. Here again, the main criteria for evaluation are the scientific importance of the research to be conducted and the necessity of the equipment for the research. Thus, the proposal should be written very much like a research proposal. It should set forth the plans and qualifications of all prospective users of the equipment or facility. If more than one research group will use it, the chances of support are better. Institutional cost-sharing is another positive factor in this type of proposal also.

There aie no application forms for NSF research grants, but there is a brochure, Grants for Scientific Research (NSF 73-12), with more detailed information about preparing and submitting proposals. Your institution's sponsored research office should have copies, or you may write to NSF, Washington, D.C. 20550. If you have questions, please write (same address) to the Division of Behavioral and Neural Sciences or to any NSF program, or telephone any of the following numbers (area code 202): Neurobiology, 634-4036; Sensory Physiology and Perception, 634-4038; Psychobiology, 6324264: Social Psychology, 632.5714; Anthropology, 632-4208; Linguistics, 632-4216. 